\title{
CCD PHOTOMETRY AND EVOLUTIONARY STATUS OF THE PULSATING VARIABLE CSS J051053.0+071722
}

\author{
K. B. Alton \\ Desert Blooms Observatory, USA. \\ Received November 28 2019; accepted January 292020
}

\begin{abstract}
Multi-color $\left(B V \mathrm{I}_{\mathrm{c}}\right)$ CCD-derived photometric data were acquired for CSS J051053.0+071722, a pulsating variable classified as a high amplitude $\delta$ Scutitype system. Analysis of precise time-series lightcurve data was accomplished using discrete Fourier transformation which revealed a mean fundamental mode $\left(f_{0}\right)$ of oscillation at $14.5097 \pm 0.0001 \mathrm{~d}^{-1}$ along with two other partial harmonics $\left(2 f_{0}\right.$ and $4 f_{0}$ ). No other statistically significant frequency shared by all bandpasses was resolved following successive pre-whitening of each residual signal. Except for 36 new times-of-maximum light produced from the present study, no other values were found in the literature; therefore, potential secular period changes could not be evaluated. The evolutionary status, age and physical nature of CSS J051053.0+071722 were investigated using the PAdova \& TRieste Stellar Evolution Code for generating stellar tracks and isochrones.
\end{abstract}

\section{RESUMEN}

Se obtuvieron datos fotométricos CCD multicolor $\left(B V \mathrm{I}_{\mathrm{c}}\right)$ para CSS J051053.0+071722, una variable pulsante clasificada como un sistema tipo $\delta$ Scuti de gran amplitud. El análisis de la curva de luz de alta precisión se realizó mediante la transformada de Fourier discreta, la cual reveló un modo fundamental medio de oscilación $\left(f_{0}\right)$ en $14.5097 \pm 0.0001 \mathrm{~d}^{-1}$, junto con otros dos armónicos parciales $\left(2 f_{0}\right.$ y $\left.4 f_{0}\right)$. No se encontró ninguna otra frecuencia apreciable compartida por todos los anchos de banda despues del pre-blanqueado sucesivo de cada señal residual. No se encontraron en la literatura datos para los tiempos de máxima luz que complementen los 36 presentados aquí. Por ello, no fue posible estudiar posibles cambios seculares del período. El estado evolutivo, la edad y la naturaleza física de CSS J051053.0+071722 se investigaron usando el código de evolución estelar PAdova\&TRieste para generar trayectorias evolutivas e isocronas.

Key Words: Hertzsprung-Russell and colour-magnitude diagrams - stars: evolution - stars: oscillations - stars: variables: Scuti

\section{INTRODUCTION}

High amplitude $\delta$ Scuti stars, hereafter HADS, constitute a very small percentage $(<1 \%)$ of all $\delta$ Sct variables (Lee et al. 2008). They commonly oscillate $(\Delta V>0.1 \mathrm{mag})$ via low-order single or double radial pulsation modes (Poretti 2003a,b; Niu et al. 2013, 2017) driven by the $\kappa$-mechanism resulting from partial ionization of He II (Pamyatnykh 1999). Many $(\approx 40 \%)$ are double pulsators showing simultaneous pulsations in the fundamental and the first overtone mode, with amplitudes generally higher in the fundamental mode (McNa- mara 2000). Non-radial pulsations have also been detected in the HADS variable V974 Oph (Poretti 2003a,b). HADS variables have historically been divided according to metallicity relative to the Sun $([\mathrm{Fe} / \mathrm{H}]=0)$. The metal-poor $([\mathrm{Fe} / \mathrm{H}]<<0)$ group is classified as SX Phe-like stars based on the prototype SX Phoenicis. Purportedly they have shorter periods $(0.02<P<0.125 \mathrm{~d})$ and lower masses $\left(\approx 1.0-1.3 M_{\odot}\right)$ than their related HADS variables possessing near solar metal abundance (McNamara 2011). SX Phe stars frequently reside in globular clusters (GC) which are ancient collections of Pop- 
ulation II stars. Therein, the majority of these pulsators are classified as blue straggler stars, paradoxically appearing much younger than their GC cohorts. Balona \& Nemec (2012) proposed that it is not possible to differentiate between $\delta$ Sct and field SX Phe variables based on pulsation amplitude, the number of pulsation modes, period or even metallicity (Garg et al. 2010). Highly sensitive space telescopes like Kepler (Gilliland et al. 2010), CoRoT (Baglin 2003) and MOST (Walker et al. 2003) have discovered many examples that contradict these traditionally accepted definitions. Balona \& Nemec (2012) further contend that the evolutionary status of each star is the only way to distinguish between these two classes.

An additional classification scheme for $\delta$ Scuti stars was recently examined by Qian et al. (2017). Therein two distinct groups of $\delta$ Scuti stars were uncovered from the LAMOST survey (Zhou et al. 2009) that fundamentally differed in effective temperature. One group was identified as normal $\delta$ Scuti stars (NDSTs) when $T_{\text {eff }}$ ranged between $6700-8500 \mathrm{~K}$ while the other was considered as unusual and cool variable stars (UCVs) with $T_{\text {eff }}$ values less than $6700 \mathrm{~K}$. A more restrictive fundamental pulsation range (0.09-0.22 d) coupled with being slightly metal poor $([\mathrm{Fe} / \mathrm{H}]=-0.25-0.0)$ further differentiates the UCVs from the NDST group. Furthermore, once the UCV stars were excluded from consideration, empirically based temperature-period, $\log g$-period, and metallicity-period relationships were developed for NDSTs.

The variability of CSS J051053.0+071722 (J2000-05:10:53.0976 +07h 17m 21.984s; $l=194.1681$ $b=-18.49118)$ was first recorded in unfiltered photometric data (also known as NSVS 9510981 and NSVS 12312737) collected during the ROTSE-I Survey (Akerlof et al. 2000). These sparsely sampled lightcurve (LC) data collected between 1999-2000 can be retrieved from the Northern Sky Variable Survey ${ }^{1}$ (Woźniak et al. 2004). More robust supporting evidence used to establish the primary oscillation period of this intrinsic variable (CSS J051053.0+071722) was gathered between 2001-2009 during the Catalina Sky Survey ${ }^{2}$ (Drake et al. 2009). Its variability was also identified from an evaluation of photometric data (also known as ASAS J051053+0717.4) collected (2005-2013) during the All Sky Automated Survey ${ }^{3}$ (Pojmanski 2000). This report marks the first multi-color

\footnotetext{
${ }^{1}$ https://skydot.lanl.gov/nsvs/nsvs.php

${ }^{2}$ http://nesssi.cacr.caltech.edu/DataRelease/

${ }^{3}$ http://www.astrouw.edu.pl/asas/?page=acvs
}

photometric study of CSS J051053.0+071722 which also describes the physical nature of this radial pulsator and critically assesses its classification as a HADS variable.

\section{OBSERVATIONS AND DATA REDUCTION}

Time-series images were acquired at Desert Blooms Observatory (DBO, USA - $110.257 \mathrm{~W}, 31.941 \mathrm{~N}$ ) with an STT-1603ME CCD camera (Diffraction Limited) mounted at the Cassegrain focus of a $0.4-\mathrm{m}$ ACF-Cassegrain telescope. This telecompressed $(0.62 \times) \mathrm{f} / 6.8$ instrument produced an image scale of $1.36 \mathrm{arcsec} / \mathrm{pixel}(\mathrm{bin}=2 \times 2)$ and a field-of-view $(\mathrm{FOV})$ of $11.6^{\prime} \times 17.3^{\prime}$. Image acquisition $(75-\mathrm{s})$ was performed using TheSkyX Pro Version 10.5.0 (Software Bisque). The CCD-camera is equipped with $B$, $V$ and $I_{c}$ filters manufactured to match the JohnsonCousins Bessell prescription. Dark subtraction, flat correction and registration of all images collected at DBO were performed with AIP 4 Win v2.4.0 (Berry \& Burnell 2005). Instrumental readings were reduced to catalog-based magnitudes using the AAVSO Photometric All-Sky Survey (APASS) star fields (Henden et al. 2009, 2010, 2011; Smith et al. 2011) built into MPO Canopus v10.7.1.3 (Minor Planet Observer). LCs for CSS J051053.0+071722 were generated using an ensemble of five non-varying comparison stars. The identity (HST Guide Star Catalog, Version GSC-ACT), J2000 coordinates and APASS color indices $(B-V)$ for these stars are provided in Table 1. Data from images taken below $30^{\circ}$ altitude (airmass $>2.0$ ) were excluded. Given that all program stars share the same FOV, differential atmospheric extinction was ignored. During each imaging session comparison stars typically stayed within $\pm 0.009 \mathrm{mag}$ for the $V$ and $I_{\mathrm{c}}$ filters and $\pm 0.018 \mathrm{mag}$ for the $B$ passband. All relevant lightcurve data (HJD, APASS magnitude, err, filter) acquired during this study at DBO can be retrieved from the AAVSO archives (https://www.aavso.org/data-download).

\section{RESULTS}

Photometric values in $B(\mathrm{n}=249), V(\mathrm{n}=262)$, and $I_{\mathrm{c}}$ $(\mathrm{n}=263)$ passbands were each processed to produce LCs that spanned $30 \mathrm{~d}$ between 25 Dec 2018 and 25 Jan 2019 (Figure 2). Time-of-maximuum (ToMax) estimates (Table 2) were derived using the polynomial extremum fit utility featured in Peranso v2.6 (Paunzen \& Vanmunster 2016). In this case a mean period solution for all passbands (0.068921 $\pm 0.000007 \mathrm{~d}$ ) was obtained using the Fourier curve fitting utility (Harris et al. 1989, FALC) featured in 
TABLE 1

ASTROMETRIC COORDINATES (J2000), $V$-mag AND COLOR INDICES $(B-V)^{*}$

\begin{tabular}{cccccc}
\hline $\begin{array}{c}\text { FOV } \\
\text { ID }\end{array}$ & GSC ID & h $\mathrm{m}$. & Dec. & APASS & APASS $^{\mathrm{a}}$ \\
$\mathrm{T}$ & $0111-02001$ & 051053.0976 & +071721.984 & 12.78 & 0.24 \\
1 & $0111-02132$ & 051059.3688 & +072142.876 & 12.02 & 0.62 \\
2 & $0111-01355$ & 051121.6096 & +071417.520 & 13.28 & 0.48 \\
3 & $0111-00631$ & 051056.5632 & +071454.780 & 12.81 & 0.66 \\
4 & $0111-00973$ & 051047.2464 & +071340.764 & 14.28 & 0.56 \\
5 & $0111-01041$ & 051022.4184 & +071531.572 & 13.06 & 0.64 \\
\hline
\end{tabular}

${ }^{*}$ For CSS J051053.0+071722 (GSC 0111-02001) and five comparison stars (1-5) used during this photometric study. ${ }^{\mathrm{a}} V$-mag and $(B-V)$ derived from APASS DR9 database.

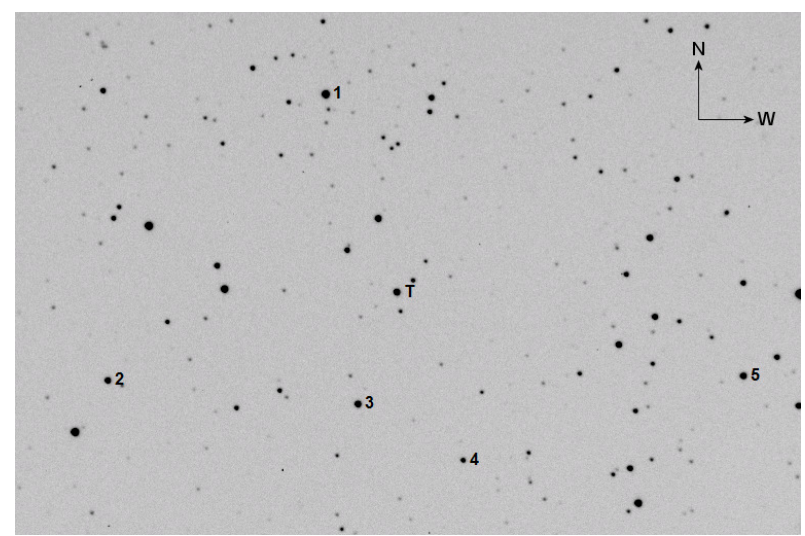

Fig. 1. FOV $(17.3 \times 11.6$ arcmin $)$ containing CSS J051053.0+071722 (T) along with the five comparison stars (1-5) used to reduce time-series images to APASS-catalog based magnitudes.

MPO Canopus v10.7.1.3. Thereafter, period determinations of survey data (NSVS, ASAS and CSS) were performed using Peranso v2.6 by applying periodic orthogonals (Schwarzenberg-Czerny 1996) to fit observations, and analysis of variance to assess fit quality. Folding together the sparsely sampled NSVS survey data revealed a period at 0.068919 $\pm 0.000024 \mathrm{~d}$ (Figure 3). The ASAS (Pojmanski 2000) photometric data acquired in 2003-2009 were period-folded and reached superimposition when $\mathrm{P}=0.068920 \pm 0.000024 \mathrm{~d}$ (Figure 4). Finally, unfiltered CCD data mined from the Catalina Sky Survey (CSS) were similarly evaluated (Figure 5) to produce a fundamental period of $\mathrm{P}=0.068919 \pm 0.000036 \mathrm{~d}$. Since no ToMax data for this variable star were found in the literature only new maxima $(n=36)$ from DBO which appear in Table 2 were used to ana-

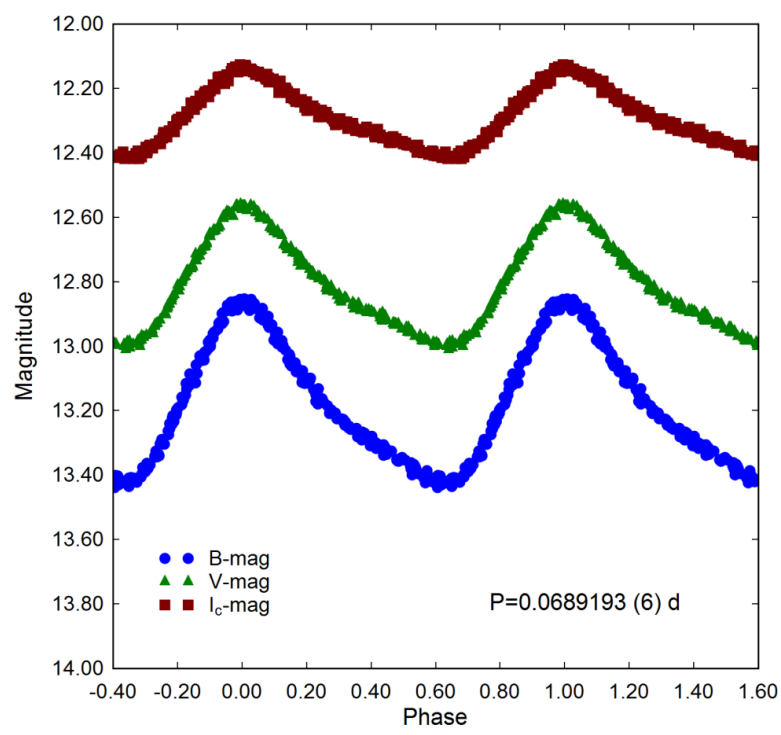

Fig. 2. Period folded (0.0689193 \pm 0.0000006 d) LCs for CSS J051053.0+071722 produced from photometric data obtained between 25 Dec 2018 and 25 Jan 2019 at DBO. LCs shown at the top $\left(I_{\mathrm{c}}\right)$, middle $(V)$ and bottom $(B)$ represent catalog-based (APASS) magnitudes determined using MPO Canopus. The color figure can be viewed online.

lyze fundamental pulse period timings (PPT). PPT differences $v s$. cycle number (Table 2) can be described by a straight line relationship from which a new linear ephemeris was calculated (equation 1):

$\operatorname{Max}(H J D)=2458508.7047(1)+0.0689193(6) E$.

These results along with the LCs from DBO (Figure 2), NSVS (Figure 3), ASAS (Figure 4) and CCS (Figure 5) also reveal that the fundamental pulsa- 


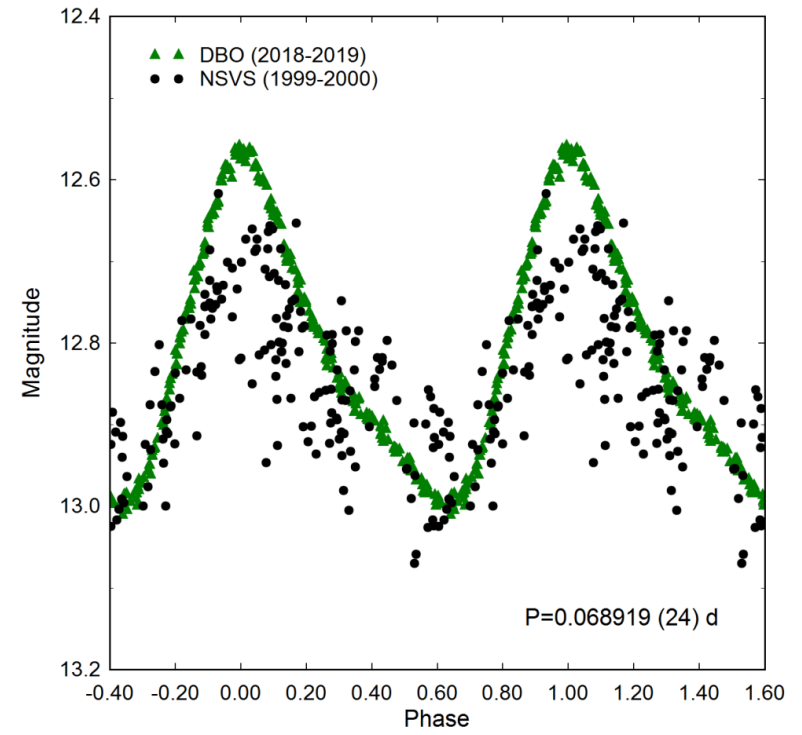

Fig. 3. Period folded (0.068919 \pm 0.000024 d) LCs for CSS J051053.0+071722 produced with sparsely sampled data (NSVS 9510981 and NSVS 12312737) from the NSVS (1999-2000) Survey. Precise time-series $V$-mag data acquired at DBO (2018-2019) are superimposed for direct comparison with ROTSE- $I$ magnitudes which have been offset to conform to the DBO-derived values. The color figure can be viewed online.

tion period has been consistent since 1999. Due to the short duration $(30 \mathrm{~d})$ of available ToMax timings no attempt was made to evaluate any underlying secular changes in period.

\subsection{Light Curve Behavior}

Morphologically, LCs of HADS variables are asymmetrical with rapid brightening to produce a sharply defined maximum peak. Thereafter a slower decline in magnitude results in a broad minimum. The largest difference between maximum and minimum light is observed in the blue passband $(\Delta B=$ $0.56 \mathrm{mag})$, followed by $V(\Delta V=0.43 \mathrm{mag})$ and finally the smallest difference detected in infrared $\left(\Delta I_{\mathrm{c}}=0.28 \mathrm{mag}\right)$. Plotting $(B-V)$ against phase (Figure 6) shows significant color amplitude $(0.125 \mathrm{mag})$ going from maximum $[(B-V) \simeq 0.3]$ to minimum light $[(B-V) \simeq 0.425]$. This behavior, commonly observed with pulsating F- to A-type stars, is more closely associated with a decrease in the effective surface temperature during minimum light.

Interstellar extinction was obtained according to Amôres \& Lépine $(2005)^{4}$ which re-

\footnotetext{
${ }^{4}$ http://www.galextin.org/v1p0/
}

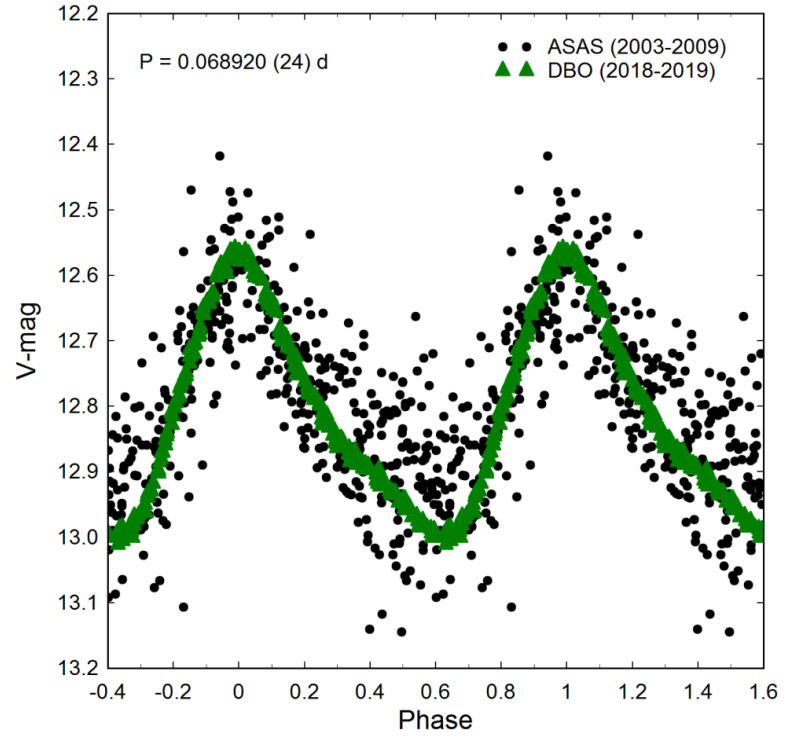

Fig. 4. Period-folded (0.068920 \pm 0.000024 d) LCs for CSS J051053.0+071722 produced with photometric data (ASAS J051053+0717.4) from the ASAS survey (20032009). Precise time-series $V$-mag data acquired at DBO (2018-2019) are superimposed for direct comparison with ASAS magnitudes ( $I$-band: Schott RG-9) which have been offset to conform to the DBO-derived values. The color figure can be viewed online.

quires galactic coordinates $(l, b)$ and distance $(\mathrm{kpc})$. Accordingly, the Model A reddening value $(E(B-V)=0.065 \pm 0.003 \mathrm{mag})$, corresponds to an intrinsic color index $(B-V)_{0}$ for CSS J051053.0+071722 that varies between $0.239 \pm 0.013 \mathrm{mag}$ at maximum light and $0.358 \pm 0.013 \mathrm{mag}$ at minimum brightness. Easy access to accurate distances from the Gaia mission (Brown et al. 2018) offers an advantage over the dust maps generated by Schlegel et al. (1998) and later adjusted by Schlafly and Finkbeiner (2011) wherein extinction is based on total dust in a given direction without regard to the target distance. This often leads to an overestimation of the reddening within the Milky Way galaxy, most commonly determined as $E(B-V)=A_{\mathrm{V}} / 3.1$. The average effective temperature $\left(T_{\text {eff }}\right)$ was estimated to be $7222 \pm 94 \mathrm{~K}$ according to the polynomial transformation equations derived by Flower (1996). These results based strictly on $(B-V)$ photometry at DBO are somewhat higher but within the uncertainty included $\left(T_{\text {eff }}=7173_{-142}^{+302} \mathrm{~K}\right)$ in the Gaia DR2 release of stellar parameters (Andrae et al. 2018). The final $T_{\text {eff }}(7178 \pm 119 \mathrm{~K})$ adopted for this study represents a mean value from 2 MASS $(7141 \pm 264 \mathrm{~K})$ using $J, K$ and $H$ transforms (Warner 2007) to 


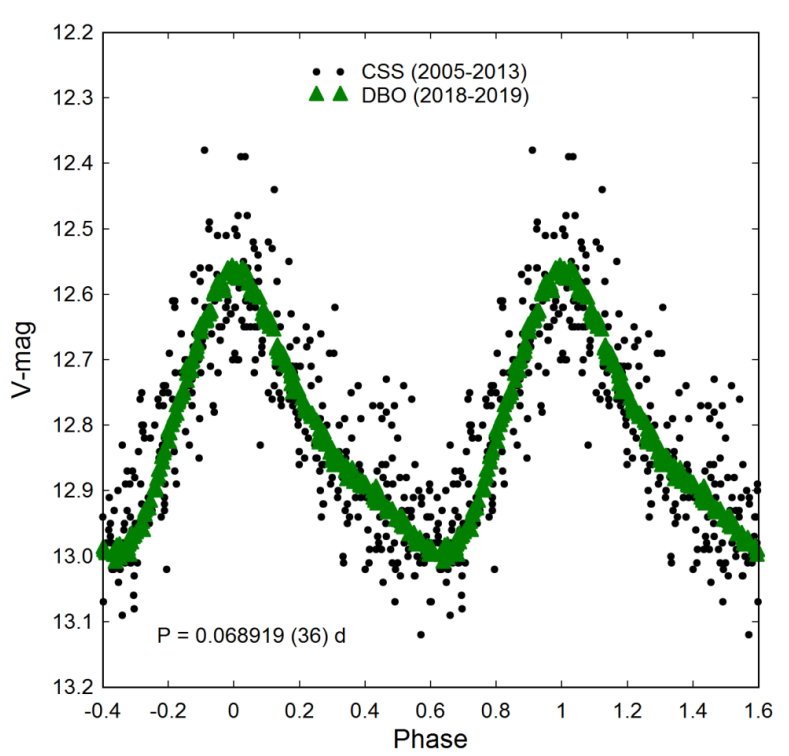

Fig. 5. Period folded (0.068919 \pm 0.000036 d) LCs for CSS J051053.0+071722 produced with unfiltered CCDderived photometric data (2005-2013) from the CSS archives. Precise time-series $V$-mag data acquired at DBO (2018) are superimposed for direct comparison with the CSS data which have been offset to conform to the DBO-derived values. The color figure can be viewed online.

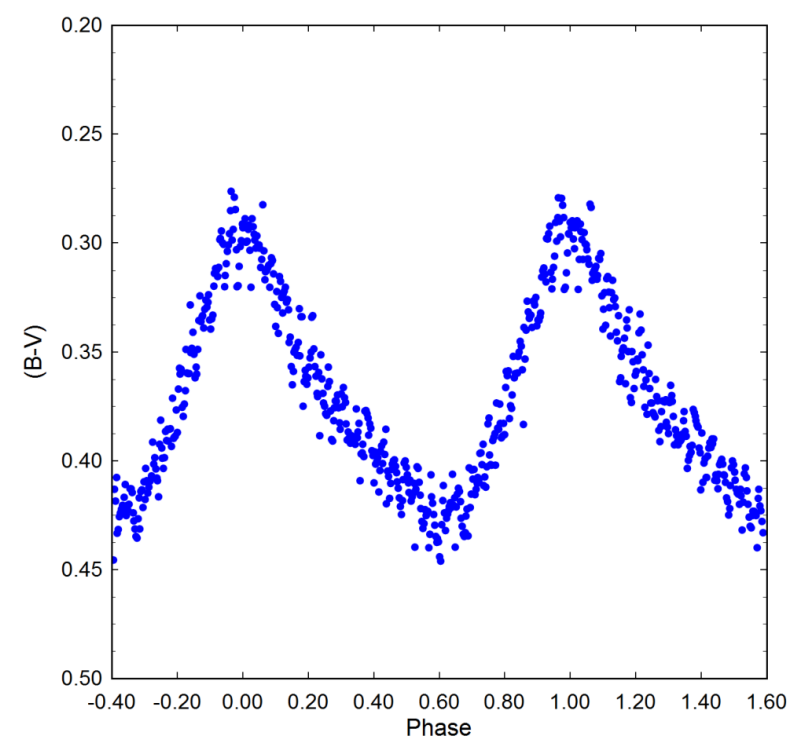

Fig. 6. CSS J051053.0+071722 LC illustrating significant color change as maximum light slowly descends to minimum light $(B-V \simeq 0.425 \mathrm{mag}$ ). This effect is most closely associated with a decrease in the effective surface temperature during minimum light. The color figure can be viewed online.
TABLE 2

TIMES OF MAXIMUM FOR
CSS J051053.0+071722*

\begin{tabular}{ccccc}
\hline ToMax $^{\mathrm{a}}$ & & & Cycle & \\
$($ HJD-2400000) & Uncertainty & Filter & No. & PTD \\
\hline 58477.6910 & 0.0006 & $V$ & 0 & 0.0000 \\
58477.6911 & 0.0006 & $B$ & 0 & 0.0001 \\
58477.6916 & 0.0013 & $I_{\mathrm{c}}$ & 0 & 0.0006 \\
58477.7597 & 0.0007 & $V$ & 1 & -0.0002 \\
58477.7598 & 0.0005 & $B$ & 1 & -0.0001 \\
58477.7603 & 0.0011 & $I_{\mathrm{c}}$ & 1 & 0.0004 \\
58477.8279 & 0.0014 & $V$ & 2 & -0.0010 \\
58477.8293 & 0.0007 & $B$ & 2 & 0.0004 \\
58477.8307 & 0.0008 & $I_{\mathrm{c}}$ & 2 & 0.0019 \\
58483.6180 & 0.0006 & $B$ & 86 & -0.0001 \\
58483.6181 & 0.0007 & $V$ & 86 & 0.0001 \\
58483.6182 & 0.0012 & $I_{\mathrm{c}}$ & 86 & 0.0002 \\
58483.6866 & 0.0007 & $V$ & 87 & -0.0004 \\
58483.6869 & 0.0005 & $B$ & 87 & -0.0001 \\
58483.6874 & 0.0012 & $I_{\mathrm{c}}$ & 87 & 0.0004 \\
58483.7558 & 0.0009 & $V$ & 88 & -0.0001 \\
58483.7560 & 0.0005 & $B$ & 88 & 0.0002 \\
58483.7566 & 0.0010 & $I_{\mathrm{c}}$ & 88 & 0.0007 \\
58483.8235 & 0.0008 & $I_{\mathrm{c}}$ & 89 & -0.0013 \\
58483.8243 & 0.0006 & $B$ & 89 & -0.0005 \\
58483.8244 & 0.0009 & $V$ & 89 & -0.0004 \\
58486.6506 & 0.0009 & $V$ & 130 & 0.0001 \\
58486.6508 & 0.0006 & $B$ & 130 & 0.0003 \\
58486.6516 & 0.0011 & $I_{\mathrm{c}}$ & 130 & 0.0011 \\
58487.6152 & 0.0008 & $V$ & 144 & -0.0001 \\
58487.6155 & 0.0005 & $B$ & 144 & 0.0001 \\
58487.6157 & 0.0008 & $I_{\mathrm{c}}$ & 144 & 0.0004 \\
58487.6837 & 0.0013 & $I_{\mathrm{c}}$ & 145 & -0.0006 \\
58487.6839 & 0.0006 & $B$ & 145 & -0.0004 \\
58487.6843 & 0.0008 & $V$ & 145 & 0.0000 \\
58508.6359 & 0.0005 & $B$ & 449 & 0.0002 \\
58508.6359 & 0.0008 & $V$ & 449 & 0.0002 \\
58508.6362 & 0.0011 & $I_{\mathrm{c}}$ & 449 & 0.0005 \\
58508.7044 & 0.0005 & $V$ & 450 & -0.0003 \\
58508.7046 & 0.0003 & $B$ & 450 & -0.0001 \\
58508.7049 & 0.0006 & $I_{\mathrm{c}}$ & 450 & 0.0003 \\
\hline
\end{tabular}

${ }^{*}$ Uncertainty, filter, epoch and fundamental pulsation timing differences (PTD) used to calculate a new linear ephemeris.

${ }^{a}$ All values acquired at DBO (2018-2019).

Johnson-Cousins, Gaia DR2 $\left(T_{\text {eff }}=7173_{-142}^{+302} \mathrm{~K}\right)$ and DBO $(7222 \pm 94 \mathrm{~K})$; the spectral type of this variable would likely range between A9 and F2. According to Qian et al. (2017), CSS J051053.0+071722 would be considered a NDST rather than a UCV since $T_{\text {eff }}$ is between 6700 and $8500 \mathrm{~K}$ while the fundamental pulsation period is less than $0.09 \mathrm{~d}$.

\subsection{Lightcurve Analysis by Discrete Fourier Transformation}

Discrete Fourier transformation (DFT) was used to extract the fundamental pulsating frequency (spectral window $=100 \mathrm{~d}^{-1}$ ) using Period04 (Lenz \& 
TABLE 3

FUNDAMENTAL FREQUENCY $\left(\mathrm{d}^{-1}\right)$ AND CORRESPONDING PARTIAL HARMONICS*

\begin{tabular}{cccccccc}
\hline & $\begin{array}{c}\text { Freq. } \\
\left(\mathrm{d}^{-1}\right)\end{array}$ & $\begin{array}{c}\text { Freq. } \\
\text { Err }\end{array}$ & $\begin{array}{c}\text { Amp. } \\
(\mathrm{mag})\end{array}$ & $\begin{array}{c}\text { Amp. } \\
\text { Err }\end{array}$ & $\begin{array}{c}\text { Phase } \\
\text { Prr }\end{array}$ & $\begin{array}{c}\text { Amp. } \\
\text { S/N }\end{array}$ \\
\hline$f_{0}-\mathrm{B}$ & 14.5097 & 0.0001 & 0.2500 & 0.0009 & 0.3563 & 0.0006 & 331 \\
$f_{0}-\mathrm{V}$ & 14.5097 & 0.0001 & 0.1919 & 0.0020 & 0.1604 & 0.0009 & 774 \\
$f_{0}-\mathrm{I}_{\mathrm{c}}$ & 14.5096 & 0.0001 & 0.1172 & 0.0006 & 0.3968 & 0.0007 & 194 \\
$2 f_{0}-\mathrm{B}$ & 29.0193 & 0.0002 & 0.0690 & 0.0007 & 0.8116 & 0.0017 & 95 \\
$2 f_{0}-\mathrm{V}$ & 29.0194 & 0.0001 & 0.0543 & 0.0024 & 0.6045 & 0.0013 & 122 \\
$2 f_{0}-\mathrm{I}_{\mathrm{c}}$ & 29.0193 & 0.0006 & 0.0339 & 0.0024 & 0.3810 & 0.0048 & 120 \\
$4 f_{0}-\mathrm{B}$ & 58.0386 & 0.0025 & 0.0065 & 0.0008 & 0.3097 & 0.0200 & 10 \\
$4 f_{0}-\mathrm{V}$ & 58.0379 & 0.3259 & 0.0039 & 0.0008 & 0.7072 & 0.1473 & 11 \\
$4 f_{0}-\mathrm{I}_{\mathrm{c}}$ & 58.3238 & 1.2568 & 0.0038 & 0.0010 & 0.1418 & 0.1428 & 7 \\
\hline
\end{tabular}

${ }^{*}$ Detected following DFT analysis of time-series photometric data $\left(B V I_{c}\right)$ from CSS J051053.0+071722.

Breger 2004). Pre-whitening steps which successively remove the previous most intense signals were employed to tease out other potential oscillations from the residuals. Only those frequencies with a $\mathrm{S} / \mathrm{N} \geq 6$ (Baran et al. 2015) in each passband are presented in Table 3. In all cases, uncertainties in frequency, amplitude, and phase were estimated by the Monte Carlo simulation $(n=400)$ routine featured in Period04. Representative amplitude spectra $\left(f_{0}\right.$, $2 f_{0}$ and $\left.4 f_{0}\right)$ from $B$-mag data acquired at DBO are shown in Figure 7 . Since the oscillation frequencies obtained from the $V$ - and $I_{\mathrm{c}}$-bandpasses are essentially redundant, they are not shown herein.

\subsection{Global Parameters}

Well over 100 years ago Henrietta Leavitt discovered a period-luminosity relationship (PLR) from Cepheid variables in the Small Magellanic Cloud (Leavitt \& Pickering 1912) and ever since pulsating stars have endured as standard candles for estimating cosmic distances. This PLR was refined afterwards to reconcile differences between metal rich (Population I) and metal-poor (Population II) Cepheids (Baade 1956). Like Cepheids, other variable stars that pulsate via the $\kappa$-mechanism were found to obey distinct PLRs. The earliest PLRs for $\delta$ Sct variables were published by Frolov (1969) and Dworak \& Zieba (1975). An improvement of the PLR for $\delta$ Sct variables was reported by McNamara (2011) albeit with Hipparcos parallaxes. A new PLR using, for the most part, more accurate values determined by the Gaia Mission (Lindegren et al. 2016; Brown et al. 2018) was recently published (Ziaali et al. 2019). Accordingly this empirically-derived expression (equation 2):

$$
M_{V}=(-2.94 \pm 0.06) \log (P)-(1.34 \pm 0.06),
$$
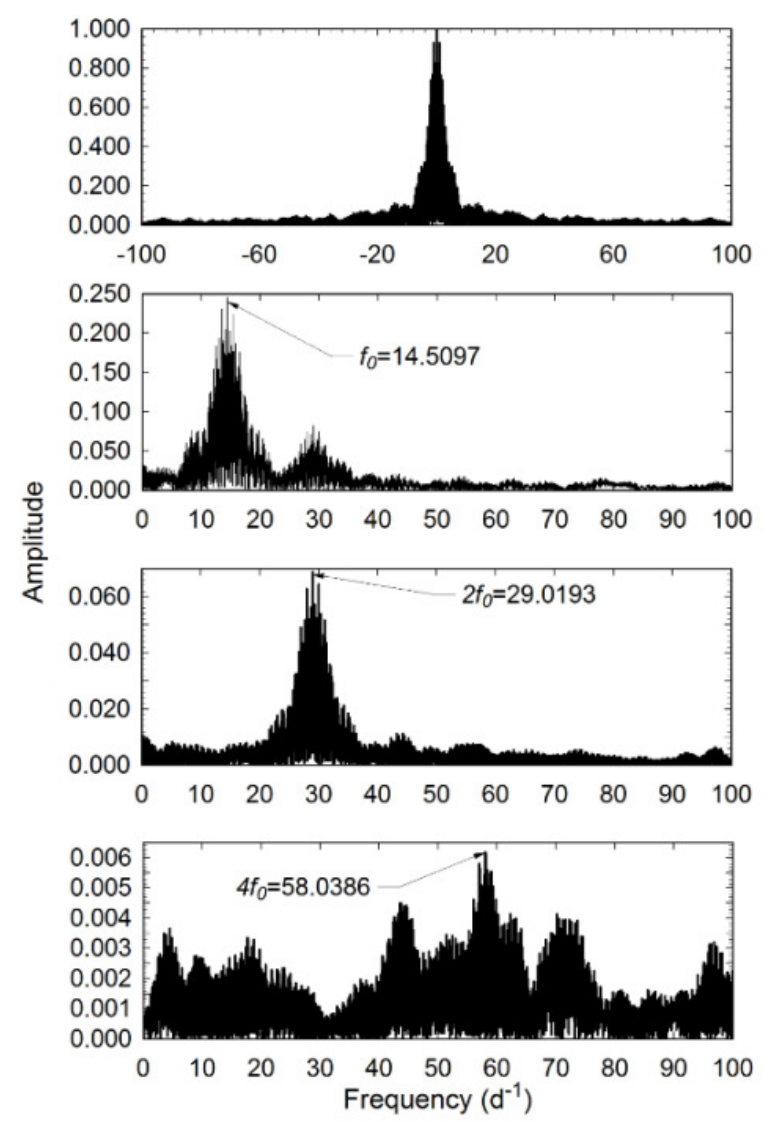

Fig. 7. Spectral window (top panel) and amplitude spectra $\left(f_{0}, 2 f_{0}\right.$ and $\left.4 f_{0}\right)$ showing all significant pulsation frequencies following DFT analysis of $B$-mag photometric data from CSS J051053.0+071722 acquired at DBO between 25 Dec 2018 and 25 Jan 2019. This includes the fundamental $f_{0}$ frequency through its highest partial harmonic $4 f_{0}$ which was detected $(\mathrm{S} / \mathrm{N} \geq 6)$ following prewhitening.

is similar to the equation published by McNamara (2011) but with improved precision.

Absolute $V_{\text {mag }}\left(M_{\mathrm{V}}\right)$ was estimated $(2.08 \pm 0.09)$ after substituting the fundamental pulsation period $(0.0689193 \mathrm{~d})$ into equation 2 . The reddening corrected distance modulus (equation 3 ):

$$
d(p c)=10^{\left.\left(m-M_{V}-A_{V}+5\right) / 5\right)}
$$

produced an estimated distance $(1263 \pm 54 \mathrm{pc})$ to CSS J051053.0+071722 using observed values for $\mathrm{m}$ $\left(V_{\text {avg }}=12.78 \pm 0.01\right)$ and $A_{\mathrm{V}}(0.200 \pm 0.008)$. By comparison, the Gaia DR2 parallax-derived distance (Bailer-Jones 2015) reported for this variable $\left(1504_{-106}^{+123}\right)$ is $16 \%$ farther. CSS J051053.0+071722 is positioned $\approx 400$ pc below the Galactic plane sug- 
gesting residence in the thick disc (Li \& Zhao 2017) rather than in the halo where many metal poor $([\mathrm{Fe} / \mathrm{H}]<-1.6)$ stars like SX Phe-type variables reside (Carollo et al. 2010).

The pulsation period and temperature/color were measured by direct observation. Similarly, the luminosity $(11.28 \pm 1.72)$ can be determined according to equation 4:

$$
L_{*} / L_{\odot}=10^{\left(\left(M_{b o l} \odot-M_{b o l *}\right) / 2.5\right)},
$$

when $M_{b o l \odot}=4.74$, and $M_{b o l *}=2.11 \pm 0.09$, where $M_{V}=2.08 \pm 0.09$ and $\mathrm{BC}=0.034$. However, the mass of an isolated field star like CSS J051053.0+071722 is very difficult to determine directly. Instead it is possible under certain conditions $\left(1.05<M_{\odot} \leq 2.40\right)$ to estimate the mass of a lone star according to a mass-luminosity relationship derived from main sequence (MS) stars in detached binary systems (Eker et al. 2018). This expression (equation 5):

$$
\log (L)=4.329( \pm 0.087) \cdot \log (M)-0.010( \pm 0.019),
$$

leads to a mass $\left(1.76 \pm 0.06 M_{\odot}\right)$. Fairly typical for a HADS variable, this result and all others derived from DBO data are summarized in Table 4. Finally, the radius in solar units $\left(R_{*}=2.17 \pm 0.21\right)$ was estimated using the well-known relationship (equation 6) where:

$$
L_{*} / L_{\odot}=\left(R_{*} / R_{\odot}\right)^{2}\left(T_{*} / T_{\odot}\right)^{4} .
$$

Derived values for the density $\left(\rho_{\odot}\right)$, surface gravity $(\log g)$, and pulsation constant $(Q)$ are also included in Table 4 . The stellar density $\left(\rho_{*}\right)$ in solar units $\left(\mathrm{g} / \mathrm{cm}^{3}\right)$ was calculated according to equation 7:

$$
\rho_{*}=3 \cdot G \cdot M_{*} \cdot m_{\odot} /\left(4 \pi\left(R_{*} \cdot r_{\odot}\right)^{3}\right),
$$

where $G$ is the $c g s$ gravitational constant, $m_{\odot}=$ solar mass $(\mathrm{g}), r_{\odot}=$ solar radius $(\mathrm{cm}), M_{*}$ is the mass and $R_{*}$ the radius of CSS J051053.0+071722 in solar units. Using the same algebraic assignments, surface gravity $(\log g)$ was determined by the following expression (equation 8):

$$
\log g=\log \left(M_{*} \cdot m_{\odot} /\left(R_{*} \cdot r_{\odot}\right)^{2}\right) .
$$

The dynamical time that it takes for a p-mode acoustic wave to internally traverse a star is strongly correlated to the stellar mean density. The pulsation constant $(Q)$ is defined by the period-density relationship (equation 9):

$$
Q=P \sqrt{\bar{\rho}_{*} / \bar{\rho}_{\odot}},
$$

TABLE 4

\section{GLOBAL STELLAR PARAMETERS FOR CSS J051053.0+071722*}

\begin{tabular}{cc}
\hline Parameter & DBO \\
\hline Mean $T_{\text {eff }}[\mathrm{K}]$ & $7178 \pm 119$ \\
Mass $\left[M_{\odot}\right]$ & $1.76 \pm 0.06$ \\
Radius $\left[R_{\odot}\right]$ & $2.17 \pm 0.21$ \\
Luminosity $\left[L_{\odot}\right]$ & $11.28 \pm 1.72$ \\
$\rho\left[\mathrm{g} / \mathrm{cm}^{3}\right]$ & $0.24 \pm 0.07$ \\
$\log \mathrm{g}[\mathrm{cgs}]$ & $4.01 \pm 0.07$ \\
$\mathrm{Q}[\mathrm{d}]$ & $0.029 \pm 0.002$ \\
\hline
\end{tabular}

${ }^{*}$ Using values determined directly from observations at DBO.

where $P$ is the pulsation period (d) and $\bar{\rho}_{*}$ and $\bar{\rho}_{\odot}$ are the mean densities of the target star and Sun, respectively. The mean density can be expressed (equation 10) in terms of other measurable stellar parameters where:

$$
\begin{gathered}
\log (Q)=-6.545+\log (P)+0.5 \log (g)+ \\
0.1 M_{\text {bol }}+\log \left(T_{\text {eff }}\right) .
\end{gathered}
$$

The full derivation of this expression can be found in Breger (1990). The resulting $Q$ value $(0.029 \pm 0.002)$ provided in Table 4 is slightly smaller than what would be expected ( $Q=0.03-0.04 \mathrm{~d}$ ) from fundamental radial pulsations observed with other $\delta$ Sct variables (Breger \& Bregman 1975; Breger 1979; Joshi \& Joshi et al. 2015; Antonello \& Pastori 2005). It is conceivable that a more extensive dataset collected at multiple sites over a much longer period of time could reveal other oscillation modes that were not detected in this study.

\section{EVOLUTIONARY STATUS OF CSS J051053.0+071722}

The evolutionary status of CSS J051053.0+071722 was evaluated (Figure 8) using the PAdova \& TRieste Stellar Evolution Code (PARSEC) for stellar tracks and isochrones (Bressan et al. 2012) and then plotted $\left(\log T_{\text {eff }}\right.$ vs. $\log \left(L / L_{\odot}\right)$ in a theoretical Hertzsprung-Russell diagram (HRD). The thick solid green-colored line defines the zero-age main sequence $(Z A M S)$ position for stars with metallicity $Z=0.014$. The two broken lines nearly perpendicular to the $Z A M S$ delimit the blue (left) and red (right) edges of the theoretical instability strip for radial low- $p$ modes (Xiong et al. 2016). Also included are 
the positions of several known HADS and SX Phetype variables (Balona 2018). The solid black circle indicates the position of CSS J051053.0+071722 using the DBO derived parameters $\left(T_{\text {eff }}\right.$ and $\left.L_{\odot}\right)$ provided in Table 4.

Interestingly a single undisputed value for the solar metallicity remains elusive. Over the last few decades, the reference metallicity values used by several authors for computing stellar models have ranged between $Z=0.012$ and 0.020 (Amard et al. 2019). Investigations still focused on finding a definitive value for $Z_{\odot}$ have been recently reported (von Steiger \& Zurbuchen 2016; Serenelli et al. 2016; Vagnozzi 2017). Despite the uncertainty in defining an absolute value for $Z_{\odot}$, an estimate for metal abundance is required in order to determine the mass, radius and age of CSS J051053.0+071722 from theoretical evolutionary tracks. A $Z$-value can be estimated indirectly from its Galactic coordinates in that its distance from the galactic plane $(-400 \mathrm{pc})$ favors a thick disc membership rather than residence in the halo where metal poor stars $([\mathrm{Fe} / \mathrm{H}]<-1.6)$ are found. Furthermore, Qian et al. (2017) report an empirical relationship between metallicity $([\mathrm{Fe} / \mathrm{H}])$ and the fundamental pulsation period $\mathrm{P}$ for an NDST star according to the following (equation 11)

$$
[\mathrm{Fe} / \mathrm{H}]=-0.121( \pm 0.026)+0.92( \pm 0.25) \times P .
$$

As expected for a thick disk resident, the predicted value $([\mathrm{Fe} / \mathrm{H}]=-0.058 \pm 0.031)$ suggests that CSS J051053.0+071722 approaches solar metallicity, or is at most a few times lower.

Two separate PARSEC evolutionary models (Bressan et al. 2012) ranging in age between $1 \times 10^{8}$ and $2.63 \times 10^{9}$ y are illustrated in Figure 8 . The red solid lines show the model tracks $\left(M_{\odot}=1.70\right.$, 1.725 and 1.75 ) over time when $Z=0.020$ while the blue, dash-dotted lines define the models $\left(M_{\odot}=1.35\right.$, 1.40 and 1.45) where $Z=0.004$. The latter simulations correspond to a decrease in metallicity by a factor of 3 to 5 depending on the reference solar metallicity. Assuming $Z=0.020$, it can be shown that CSS J051053.0+071722 would have a mass of $1.71 \pm 0.03 M_{\odot}$ and a radius of $2.15 \pm 0.06 R_{\odot}$. The position of this intrinsic variable near the $M_{\odot}=1.70$ evolutionary track extrapolates to an age of $1.24 \pm 0.05$ Gyr suggesting it is a moderately evolved MS object lying amongst other HADs closer to the blue edge of the instability strip.

By comparison, if CSS J051053.0+071722 is a metal poor $(Z=0.004)$ star, then it would have a somewhat greater radius $\left(2.44 \pm 0.02 R_{\odot}\right)$, but

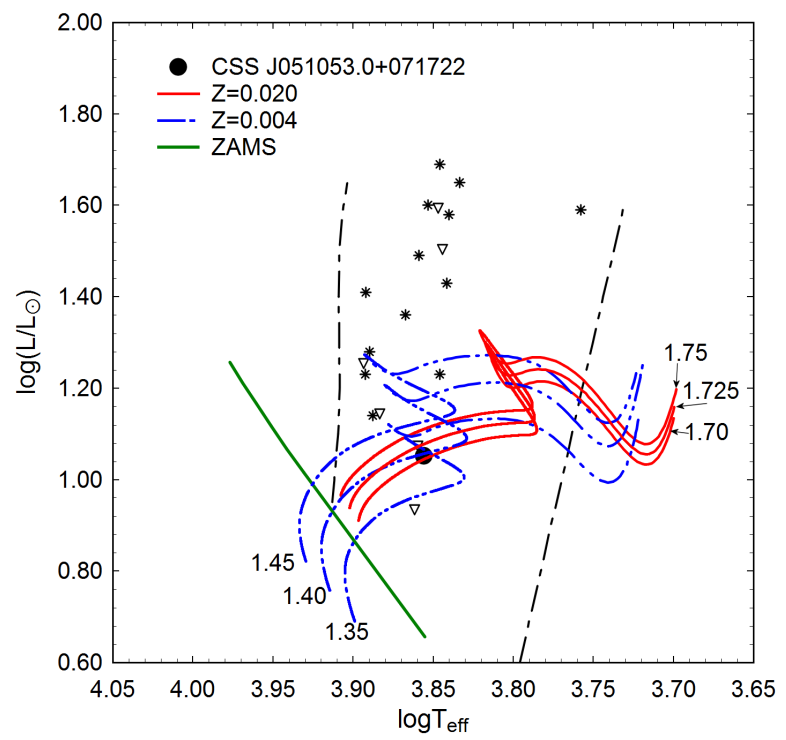

Fig. 8. Evolutionary tracks (red solid lines; $Z=0.020$ and blue dashed lines; $Z=0.004)$ derived from PARSEC models (Bressan et al. 2012) showing position of CSS J051053.0+071722 (black filled circle) relative to $Z A M S$ (thick green line) and within the theoretical instability strip (black dashed lines) for low-order radial mode $\delta$ Scuti pulsators. The position of other HADS $\left(^{*}\right)$ and SX Phe (open triangle) variables reported by Balona (2018) are included for comparison. The color figure can be viewed online.

would be less massive $\left(1.40 \pm 0.02 M_{\odot}\right)$. Its position very close to the $1.4 M_{\odot}$ track lies prior to the HRD region where evolutionary tracks of low metallicity stars begin stellar contraction near the end of hydrogen burning in the core. This star would still be a MS object but with an age approaching $2.44 \pm 01$ Gyr. Alternatively, an even less massive and smaller $\left(R_{*}<2.21 R_{\odot}\right)$ star positioned slightly higher than the $1.35 M_{\odot}$ trajectory would be found inside the HRD region where the evolutionary tracks of low metallicity stars begin to zigzag due to stellar contraction. Based on this scenario the age of CSS J051053.0+071722 would approach 2.55 Gyr.

In summary, the theoretical mass $\left(1.71 \pm 0.03 M_{\odot}\right)$ where $\mathrm{Z}=0.020$ favors the higher metallicity of CSS J051053.0+071722 which is also in good agreement with results $\left(1.76 \pm 0.06 M_{\odot}\right)$ independently determined using an empirical massluminosity relationship. If or when high resolution spectroscopic data become available in the future, uncertainty about the mass and metallicity of CSS J051053.0+071722 will likely decrease. 


\section{CONCLUSIONS}

This first multi-color $\left(B V I_{\mathrm{c}}\right) \quad \mathrm{CCD}$ study of CSS J051053.0+071722 has produced 36 new timesof-maximum. Due to the short duration and lack of other published ToMax values, no attempt was made to evaluate secular changes in the fundamental mode of oscillation. Deconvolution of timeseries photometric data by discrete Fourier transformation shows that this star is a monoperiodic radial pulsator $\left(f_{0}=14.5097 \mathrm{~d}^{-1}\right)$ which also oscillates in at least 2 other partial harmonics $\left(2 f_{0}\right.$ and $\left.4 f_{0}\right)$. A mean effective temperature for CSS J051053.0+071722 (7178 $\pm 119 \mathrm{~K})$ was estimated from a composite of Gaia DR2, 2MASS and DBO results which likely corresponds to spectral type A9-F2. The pulsation period $(\approx 0.068919 \mathrm{~d})$, radial oscillation mode, $V_{\text {mag }}$ amplitude $(0.43 \mathrm{mag})$, spectral type and LC morphology are all consistent with the defining characteristics of a HADS variable. It should be noted that these attributes do not necessarily exclude the possibility that CSS J051053.0+071722 is a field SX Phe-type variable. However, the generally accepted threshold for SX Phe stars is <1.3 $M_{\odot}$ (McNamara 2011) which in this case is far less than the mass predicted from a $M-L$ relationship $\left(\approx 1.76 M_{\odot}\right)$ and evolutionary modeling $\left(\approx 1.71 M_{\odot}\right)$. Given these results, the weight of evidence confirms the classification of CSS J051053.0+071722 as a HADS variable.

This research has made use of the SIMBAD database operated at Centre de Données astronomiques de Strasbourg, France. In addition, the Northern Sky Variability Survey hosted by the Los Alamos National Laboratory, the All Sky Automated Survey Catalogue of Variable Stars and the Catalina Surveys Data Release 2 archives were mined for essential information. This work also presents results from the European Space Agency (ESA) space mission Gaia. Gaia data are being processed by the Gaia Data Processing and Analysis Consortium (DPAC). Funding for the DPAC is provided by national institutions, in particular the institutions participating in the Gaia MultiLateral Agreement (MLA). The Gaia mission website is https://www.cosmos.esa.int/gaia while the Gaia archive website is https://archives.esac.esa.int/gaia. The careful review and helpful commentary provided by an anonymous referee is gratefully acknowledged.

\section{REFERENCES}

Akerlof, C., Amrose, S., Balsano, R., et al. 2000, PASP, 122,131
Amard, L., Palacios, A., Charbonnel, C., et al. 2019, A\&A, 631, A77

Amôres, E. B. \& Lépine, J. R. D. 2005, AJ, 130, 659

Andrae, R., Fouesneau, M., Creevey, O., et al. 2018, $\mathrm{A} \& \mathrm{~A}, 616, \mathrm{~A} 8$

Antonello, E. \& Pastori, L. 1981, PASP, 93, 237

Baade, W. 1956, PASP, 68, 5

Baglin, A. 2003, AdSpR, 31, 345

Bailer-Jones, C. A. L. 2015, PASP, 127, 994

Balona, L. A. \& Nemec, J. M. 2012, MNRAS, 426, 2413

Balona, L. A. 2018, MNRAS, 479, 183

Baran, A. S., Koen, C., \& Porkrzywka, B. 2015, MNRAS, 448, L16

Berry, R. \& Burnell, J. 2005, The Handbook of Astronomical Image Processing, Wilmann-Bell, Inc., Richmond, VA

Breger, M. 1979, PASP, 91, 5 . 1990, DSSN, 1, 13

Breger, M. \& Bregman, J. N. 1975, ApJ, 200, 343

Bressan, A., Marigo, P., Giradi, L., G., et al. 2012, MNRAS, 427, 127

Brown, A. G. A., Vallenari, A., Prusti, T., et al. 2018, A\&A, 616, A1

Carollo, D., Beers, T. C., Chiba, M., et al. 2010 ApJ, 712,692

Drake, A. J., Djorgovski, S. G. Mahabal, A., et al. 2009, ApJ, 696, 870

Dworak, T. Z. \& Zieba, S. 1975, IBVS, 1005, 1

Eker, Z., Bakış, V., Bilir, S., et al. 2018, MNRAS, 479, 5491

Flower, P. J. 1996, ApJ, 469, 355

Frolov, M. S. 1969, ATsir, 505, 1

Garg, A., Cook, K. H., Nikolaev, S., et al. 2010, AJ, 140, 328

Gilliland, R. L., Brown, T. M., Christensen-Dalsgaard, J., et al. 2010, PASP, 122, 131

Harris, A. W., Young, J. W., Bowell, E., et al. 1989, Icar, 77,171

Henden, A. A., Welch, D. L., Terrell, D., \& Levine, S. E. 2009, AAS, 41, 669

Henden, A. A., Terrell, D., Welch, D. L., \& Smith, T. C. 2010, American Astronomical Society Meeting Abstracts, 42, 515

Henden, A. A., Levine, S. E., Terrell, D., et al. 2011, American Astronomical Society Meeting Abstracts, 43, 2011

Joshi, S. \& Joshi, Y. C. 2015, JApA, 36, 33

Leavitt, H. S. \& Pickering, E. C. 1912, Harvard College Observatory Circular

Lee, Y.-H, Kim, S. S., Shin, J., et al. 2008, PASJ, 60, 3

Lenz, P. \& Breger, M. 2004, IAUS, 224, 786

Li, C. \& Zhao, G. 2017, ApJ, 850, 25

Lindegren, L., Lammers, U., Bastian, U., et al. 2016, A\&A, 595, A4

McNamara, D. H. 2000, ASPC, 210, 373

McNamara, D. H. 2011, AJ, 142, 110

Niu, J. S., Fu, J.-N., \& Zong, W.-K. 2013, RAA, 13, 1181 
Niu, J. S., Fu, J.-N., Li, Y., et al. 2017, MNRAS, 467, 3122

Pamyatnykh, A. A. 1999, AcA, 49, 119

Paunzen, E. \& Vanmunster, T. 2016, AN, 337, 239

Pojmanski, G. 2000, AcA, 50, 177

Poretti, E. 2003a, A\&A, 409, 1031 2003b, ASPC, 292, 145

Qian, S.-B., Li, L.-J., He, J.-J., et al. 2017, MNRAS, 475, 478

Schwarzenberg-Czerny, A. 1996, ApJ, 460, L107

Schlafly, E. F. \& Finkbeiner, D. P. 2011, ApJ, 737, 103

Serenelli, A., Scott, P., Villante, F. L., et al. 2016, MNRAS, 463, 2

Smith, T. C., Henden, A. A., \& Starkey, D. R. 2011, SASAS, 30, 121
Vagnozzi, S., Freese, K. \& Zurbuchen, T. H. 2017, ApJ, 839,55

von Steiger, R. \& Zurbuchen, T. H. 2016, ApJ, 816, 13

Walker, G., Matthews, J., Kuschnig, R., et al. 2003, PASJ, 115, 1023

Warner, B. D. 2007, Minor Planet Bulletin, 34, 113

Woźniak, P. R., Vestrand, W. T., Akerlof, C. W., et al. 2004, AJ, 127, 2436

Xiong, D. R., Deng, L., Zhang, C., \& Wang, K. 2016, MNRAS, 457, 3163

Zhou, A.-Y., Jiang, X.-J., Zhang, Y. P., \& Wei, J.-Y. 2009, RAA, 9, 349

Ziaali, E., Bedding, T. R., Murphy, S. J., et al. 2019, MNRAS, 486, 4348

Kevin B. Alton: UnderOak and Desert Blooms Observatories, 70 Summit Ave., Cedar Knolls, NJ, USA (mail@underoakobservatory.com). 